PROCEEDINGS OF THE

AMERICAN MATHEMATICAL SOCIETY

Volume 35, No. 1, September 1972

\title{
SPARSE SUBSETS OF ORTHONORMAL SYSTEMS
}

\author{
J. J. PRICE
}

\begin{abstract}
There exist families of Walsh, Haar and trigonometric functions that have density zero and yet are complete in the sense of measure.
\end{abstract}

1. Introduction. Goffman and Waterman proved elegantly [1] that from a system of functions which is total in measure on $[0,1]$, one may discard a suitable infinite subset without destroying totality. It is natural to ask "how many" functions may be discarded from specific systems or equivalently, how "thin" a subset can be and still be total in measure. In this regard, we have proved [2] that a system $\left\{\psi_{n_{i}}\right\}$ of Walsh functions is total in measure on $[0,1]$ if it has density 1 , where

$$
\text { density }=\limsup _{k} \limsup _{n} \frac{\Lambda(n+k)-\Lambda(n)}{k},
$$

$\Lambda(n)$ denoting the number of indices $n_{i}$ for which $n_{i} \leqq n$.

In this paper we show there are actually Walsh families of density zero that are total in measure on $[0,1]$, and that the same is true for the trigonometric and Haar functions:

THEOREM. There exist subsets of density zero of the Walsh functions, the trigonometric functions $\{\cos 2 \pi n x, \sin 2 \pi n x\}$, and the Haar functions that are total in measure on $[0,1]$.

We shall give separate proofs for the three systems in the next three sections.

Notation. If $\Phi$ is a set of functions, $L(\Phi)$ will denote the set of all finite real linear combinations of elements of $\Phi$. The word "total" will always mean "total in measure".

2. Walsh functions. We use a well-known property of the Dirichlet kernels of the Walsh system:

$$
\begin{aligned}
D_{n}(x)=1+\sum_{j=1}^{2^{n}-1} \psi_{j}(x) & =2^{n}, & & x \in\left[0,2^{-n}\right), \\
& =0, & & x \in\left[2^{-n}, 1\right) .
\end{aligned}
$$

Received by the editors October 6, 1971.

AMS 1970 subject classifications. Primary 42A52; Secondary 28A20.

Key words and phrases. Walsh functions, Haar functions, total in measure.

1. American Mathematical Society 1972 
If $g_{n}(x)=1-D_{n}(x)$, then

$$
\begin{aligned}
g_{n}(x)=-\sum_{j=1}^{2^{n}-1} \psi_{j}(x) & =1-2^{n}, & & x \in\left[0,2^{-n}\right), \\
& =1, & & x \in\left[2^{-n}, 1\right) .
\end{aligned}
$$

Clearly $g_{n}(x) \rightarrow 1$ in measure on $[0,1]$.

We define

$$
f_{l \prime}(x)=g_{n}\left(2^{n^{2}} x\right)=\sum_{j=1}^{2^{n}-1} \psi_{j}\left(2^{n^{2}} x\right) .
$$

Then $f_{n}(x) \rightarrow 1$ in measure on $[0,1]$. Furthermore, since for any Walsh function $\psi_{j}(x), \psi_{j}\left(2^{k} x\right)=\psi_{j 2^{k}}(x)$, we have $f_{n} \in L\left(\Psi_{n}\right)$ where

$$
\Psi_{n}=\left[\psi_{k}: k=j \cdot 2^{n^{2}}, j=1,2, \cdots, 2^{n}-1\right] .
$$

Now let $\varphi_{1}, \varphi_{2}, \varphi_{3}, \cdots$ denote the sequence of Walsh functions $\psi_{0}, \psi_{1}$, $\psi_{0}, \psi_{1}, \psi_{2}, \cdots$ in which each Walsh function occurs infinitely often. Define $\Psi=\bigcup_{n=1}^{\infty} \varphi_{n} \Psi_{n}$. The set $\varphi_{n} \Psi_{n}$ consists of Walsh functions whose indices are in arithmetic progression, with common difference $2^{n^{2}}$. It follows that $\Psi$ has density zero. To see that $\Psi$ is total, note that for each Walsh function $\psi_{k}$, there is a sequence $\left\{n_{i}\right\}$, depending on $k$, for which $\psi_{k} \Psi_{n_{i}} \subset \Psi$. Therefore,

$$
\psi_{k} f_{n_{i}} \in L\left(\psi_{k} \Psi_{n_{i}}\right) \subset L(\Psi)
$$

But $f_{n_{i}}(x) \rightarrow 1$, so $\psi_{k}(x) f_{n_{i}}(x) \rightarrow \psi_{k}(x)$ in measure on $[0,1]$. Thus each Walsh function can be approximated arbitrarily closely in measure by elements of $L(\Psi)$. Therefore $\Psi$ is total.

3. Trigonometric functions. As in the preceding section, we construct a sequence $\left\{f_{n}(x)\right\}$ such that $f_{n}(x) \rightarrow 1$ in measure on $[0,1]$. This time we use the fact that $K_{n}(x) \rightarrow 0$ in measure on $[0,1]$, where $K_{n}(x)$ is the Fejer kernel.

Let $\left\{m_{n}\right\}$ be a sequence of positive integers increasing so rapidly that $m_{n+1}>m_{n}^{2}$, for instance $m_{n}=2^{n !}$. Define

$$
f_{n}(x)=1-K_{m_{n}}\left(m_{n} x\right)=\sum_{k=1}^{m_{n}} a_{k n} \cos \left(2 \pi m_{n} k x\right) .
$$

Then $f_{n}(x) \rightarrow 1$ in measure on $[0,1]$ and $f_{n} \in L\left(\Phi_{n}\right)$ where

$$
\Phi_{n}=\left[\cos 2 \pi j x: j=m_{n}, 2 m_{n}, \cdots, m_{n}^{2}\right] .
$$

Let $\left\{k_{n}\right\}$ denote the sequence $0,1,0,1,2,0,1,2,3, \cdots$. Define

$$
\Phi_{n}^{\prime}=\left[\sin 2 \pi j x, \cos 2 \pi j x: j=m_{n} \pm k_{n}, 2 m_{n} \pm k_{n}, \cdots, m_{n}^{2} \pm k_{n}\right]
$$

and $\Phi=\bigcup_{n=1}^{\infty} \Phi_{n}^{\prime}$. It is clear that $\Phi$ has density zero. 
For each positive integer $j$, there is a sequence $\left\{n_{i}\right\}$ depending on $j$, such that

$$
(\sin 2 \pi j x) \Phi_{n_{i}} \in L\left(\Phi_{n_{i}}^{\prime}\right), \quad(\cos 2 \pi j x) \Phi_{n_{i}} \in L\left(\Phi_{n_{i}}^{\prime}\right) .
$$

It follows that

$$
(\sin 2 \pi j x) f_{n_{i}}(x),(\cos 2 \pi j x) f_{n_{i}}(x) \in L\left(\Phi_{n_{i}}^{\prime}\right) \subset L(\Phi) .
$$

Since $f_{n_{i}}(x) \rightarrow 1$ in measure on $[0,1]$,

$$
(\sin 2 \pi j x) f_{n_{i}}(x) \rightarrow \sin 2 \pi j x, \quad(\cos 2 \pi j x) f_{n_{i}}(x) \rightarrow \cos 2 \pi j x .
$$

Hence each function $\sin 2 \pi j x, \cos 2 \pi j x$ can be approximated arbitrarily closely in measure by elements of $L(\Phi)$. Therefore $\Phi$ is total.

4. Haar functions. Here we use a criterion proved by Robert E. Zink and the author [3]: Suppose $\left\{h_{j}\right\}$ is a family of Haar functions. Let $\sigma\left(h_{j}\right)$ denote the support of $h_{j}$ and let $E=\lim \sup \sigma\left(h_{j}\right)$. Then $\left\{h_{j}\right\}$ is total on a set $G \subset[0,1]$ if and only if $|G|=|G \cap E|$. In view of this criterion we shall exhibit a subset $H$ of the Haar functions with density zero and such that $|E|=1$.

Let $B_{n}$ denote the block of Haar functions $\left[h_{j}: 2^{n} \leqq j<2^{n+1}\right]$. For each positive integer $k$, let $G_{k}$ be the union of any collection of $2^{k}$ blocks $B_{n}$, subject to two conditions:

(a) If $B_{n}, B_{m} \in G_{k}$, then $|n-m|>k$.

(b) If $B_{n} \in G_{k}$ and $B_{m} \in G_{k+1}$, then $n<m$.

(These conditions imply $n>2^{k}$ when $B_{n} \in G_{k}$ and $k>2$.)

Now we define the desired system $H$ of Haar functions. For each $k$ let $H_{k}=\left[h_{j}: h_{j} \in G_{k}\right.$ and $\left.n \equiv 0\left(\bmod 2^{k}\right)\right]$; then set $H=\bigcup_{k=1}^{\infty} H_{k}$. Clearly $H$ has density zero (just as $\Psi$ has density zero in $\S 2$ ).

For each $B_{n}$ that occurs in some $G_{k}$, let

$$
E_{n}=\bigcup \sigma\left(h_{j}\right) \quad\left(h_{j} \in B_{n} \cap H_{k}\right) .
$$

It is not hard to see that

$$
E_{n}=\left[x: d_{i}(x)=0, n-k+1 \leqq i \leqq n\right]
$$

where $d_{i}(x)$ is the $i$ th dyadic digit of $x$. Thus $\left|E_{n}\right|=2^{-k}$. Because of (a) and (b), each $E_{n}$ is defined by conditions on different sets of dyadic digits, hence the $E_{n}$ are independent sets. Since there are $2^{k}$ sets $E_{n}$ for each $k$,

$$
\sum\left|E_{n}\right|=\sum_{k} \sum_{B_{n} \in G_{i k}}\left|E_{n}\right|=\sum_{k}\left(2^{k} \cdot 2^{-k}\right)=\sum 1=\infty .
$$

It follows by the Borel-Cantelli Lemma that $\left|\lim \sup E_{n}\right|=1$ which implies that $H$ is total on $[0,1]$. 


\section{REFERENCES}

1. C. Goffman and D. Waterman, Basic sequences in the space of measurable functions, Proc. Amer. Math. Soc. 11 (1960), 211-213. MR 22 \#2886.

2. J. J. Price, A density theorem for Walsh functions, Proc. Amer. Math. Soc. 18 (1967), 209-211. MR 35 \#656.

3. J. J. Price and R. E. Zink, On sets of completeness for families of Haar functions, Trans. Amer. Math. Soc. 119 (1965), 262-269. MR 32 \#1499.

Department of Mathematics, University of Paris, Orsay, France

Department of Mathematics, Purdue University, West Lafayette, Indiana 47907 